\title{
RELAÇÃO ENTRE CEFALOMETRIA E ANÁLISE FACIAL NA DETERMINAÇÃO DO TIPO DE FACE
}

\author{
Relation between cephalometry and facial analysis \\ on the facial type determination
}

\author{
Rossana Ribeiro Ramires (1), Léslie Piccolotto Ferreira (2), Irene Queiroz Marchesan ${ }^{(3)}$, \\ Débora Martins Cattoni ${ }^{(4)}$, Marta Assumpção de Andrada e Silva ${ }^{(5)}$
}

\begin{abstract}
RESUMO
Objetivo: correlacionar os achados cefalométricos com os da análise facial realizada por meio de fotografias para classificar o tipo facial, segundo o sexo. Métodos: participaram 105 adultos leucodermas, 34 (32,4\%) homens e 71 (67,6\%) mulheres, com idades entre 20 e 40 anos, de uma clínica particular de ortodontia de Belo Horizonte/MG. Os achados da análise cefalométrica para determinação do tipo facial a partir do índice VERT de Ricketts foram comparados com a classificação da face realizada por meio da análise facial clínica baseada na observação de duas fotografias. Para essa análise, participaram três fonoaudiólogos especialistas em motricidade orofacial. Resultados: não houve correspondência satisfatória ao se comparar as duas formas de classificação do tipo facial apresentadas neste estudo. A maioria dos indivíduos masculinos dolicofaciais foi classificada como face média a partir da análise facial, ou seja, quatro entre sete sujeitos $(57,14 \%)$. Os 11 mesofaciais foram identificados como face média $(\mathrm{N}=5 ; 45,45 \%)$ ou curta $(\mathrm{N}=4 ; 36,36 \%)$ e os 16 braquifaciais como face média $(\mathrm{N}=9 ; 56,25 \%)$ ou curta $(\mathrm{N}=7 ; 43,75 \%)$. No sexo feminino, observou-se que 11 entre 20 , ou seja, $55 \%$ dos indivíduos dolicofaciais foram apontados como face média. Dos 26 sujeitos mesofaciais, $16(61,53 \%)$ também foram classificados como face média. Com relação aos 25 braquifaciais, 12 (48\%) foram identificados como face curta e 11 (44\%), como média. Conclusão: a classificação do tipo de face apenas a partir da análise facial realizada por meio de fotografias não foi considerada confiável, se utilizada isoladamente, quando comparada à classificação obtida a partir da análise cefalométrica.
\end{abstract}

DESCRITORES: Face; Morfologia; Adulto; Circunferência Craniana; Fotografia; Estudo Comparativo

(1) Fonoaudióloga; Mestre em Fonoaudiologia pela Pontifícia Universidade Católica de São Paulo.

(2) Fonoaudióloga; Professora Titular da Graduação e PósGraduação em Fonoaudiologia da Pontifícia Universidade Católica de São Paulo, PUCSP, São Paulo, SP; Coordenadora e docente do Curso de Especialização em Fonoaudiologia - Voz da Coordenadoria Geral de Especialização, Aperfeiçoamento e Extensão da Pontifícia Universidade Católica de São Paulo, COGEAE-PUC/SP, São Paulo, SP; Doutora em Distúrbios da Comunicação Humana.

(3) Fonoaudióloga; Diretora Clínica do CEFAC - Pós-Graduação em Saúde e Educação; Doutora em Educação pela Universidade Estadual de Campinas.

(4) Fonoaudióloga; Professora do CEFAC - Pós-Graduação em Saúde e Educação; Doutora em Ciências pela Faculdade de Medicina da Universidade de São Paulo.

(5) Fonoaudióloga; Professora Assistente Doutora da Graduação e Pós-Graduação em Fonoaudiologia da Pontifícia Universidade Católica de São Paulo, PUCSP, São Paulo, SP; Professora Adjunta no Curso de Fonoaudiologia da

\section{INTRODUÇÃO}

A cefalometria é uma técnica radiográfica a partir da qual são obtidos dados referentes ao desenvolvimento craniofacial, tipo de oclusão, tipologia facial, dentre outros ${ }^{1}$. Tem como objetivos principais a avaliação do crescimento, o auxílio na determinação do diagnóstico ortopédico facial, ortodôntico e

Faculdade de Ciências Médicas da Irmandade da Santa Casa de Misericórdia de São Paulo, FCM-ISCMSP, São Paulo, SP; Professora nos Cursos de Especialização em Voz da Coordenadoria Geral de Especialização, Aperfeiçoamento e Extensão da Pontifícia Universidade Católica de São Paulo, COGEAE-PUC/SP, São Paulo, SP; Professora do CEFAC - Pós-Graduação em Fonoaudiologia e Educação; Doutora em Comunicação e Semiótica pela Pontifícia Universidade Católica de São Paulo.

Conflito de interesses: inexistente 
cirúrgico, além de contribuir para o planejamento do tratamento tanto nas etapas iniciais como intermediárias ${ }^{2,3}$. Esse exame também é importante para diagnóstico e acompanhamento fonoaudiológico de pacientes que apresentam alterações miofuncionais orofaciais ${ }^{1}$.

A análise da face, outro instrumento fundamental de diagnóstico para ortodontistas, cirurgiões bucomaxilofaciais ${ }^{4}$, fonoaudiólogos e profissionais afins, pode ser feita de forma direta ou indireta. A análise direta ou tridimensional, por meio do exame clínico, promove uma avaliação mais satisfatória das estruturas orofaciais se comparada à forma indireta caracterizada pela análise bidimensional realizada por meio de cefalometria ou fotografias ${ }^{5,6}$. Apesar da análise direta ser mais satisfatória, a utilização de fotografias da face pelo clínico é considerada uma importante ferramenta para se estabelecer 0 diagnóstico, desde que elas sejam padronizadas e de boa qualidade ${ }^{7,8}$.

Para a determinação do tipo facial, que é a variação do esqueleto craniofacial, a utilização da cefalometria é soberana. Entretanto, não é um exame solicitado pelo fonoaudiólogo e sim pelo ortodontista. Observa-se na prática clínica que, muitas vezes, o fonoaudiólogo apenas analisa visualmente a face do paciente e define sob a sua óptica e conhecimento qual o tipo de face se apresenta diante das características ósseas, musculares e funcionais.

O objetivo desta pesquisa foi correlacionar os achados cefalométricos com os da análise facial realizada por meio de fotografias para classificar 0 tipo facial, segundo o sexo.

\section{MÉTODOS}

Participaram da pesquisa 105 adultos leucodermas, $34(32,4 \%)$ homens e $71(67,6 \%)$ mulheres, com idades entre 20 e 40 anos, de uma clínica particular de ortodontia de Belo Horizonte/ MG.

Adotou-se como critério de inclusão possuir documentação ortodôntica com análise cefalométrica de Ricketts. Como critérios de exclusão foram adotados: apresentar histórico de cirurgia ortognática e ser da raça negra ou oriental. Não foi levado em consideração o fato dos pesquisados se encontrarem em tratamento ortodôntico ou o tipo de oclusão dentária apresentada.

A determinação do tipo facial por meio da cefalometria foi a partir do cálculo do índice VERT de Ricketts, baseado em cinco grandezas cefalométricas (ângulo do eixo facial, profundidade facial, ângulo do plano mandibular, altura facial inferior e arco mandibular) ${ }^{9}$. Os sujeitos foram classificados como dolicofacial (índice VERT menor que -0,5), mesofacial (valores entre $-0,5$ e $+0,5$ ) ou braquifacial (VERT maior que 0,5 ).

Duas fotografias dos participantes, uma da face frontal e outra de perfil direito, ambas sem sorriso, que constavam da pasta de documentação ortodôntica, foram digitalizadas pela pesquisadora e gravadas em um compact disk (CD). A ordem dos participantes seguiu a sequência em que ocorreu a coleta dos dados, o que significa que os tipos faciais estavam dispostos de forma aleatória.

Trêsjuízes representadosporfonoaudiólogos(as) especialistas em motricidade orofacial, com experiência mínima de cinco anos, participaram da pesquisa. Foi agendada uma data prévia para sua participação. Os juízes foram treinados juntos e simultaneamente pela pesquisadora e tiraram suas dúvidas com a realização de um teste com cinco pesquisados que não foram incluídos no estudo. Foram orientados que poderiam ver as fotos de cada sujeito quantas vezes desejassem. Caso tivessem dúvidas, podiam rever as imagens de quem quisessem. As respostas sobre o tipo facial de cada sujeito foram obtidas por consenso, ou seja, por uma decisão conjunta após discussão de cada caso. Os juízes classificaram as faces em longa, média ou curta.

Todos os sujeitos realizaram a documentação ortodôntica em um dos dois laboratórios que prestavam serviços para a clínica e seguiam a mesma padronização tanto para a análise cefalométrica, como para as fotografias tiradas.

A pesquisa foi aprovada pelo Comitê de Ética da Pontifícia Universidade Católica de São Paulo, sob o número 0021/2006. Todos os participantes do estudo, inclusive os juízes, leram e assinaram o termo de consentimento livre e esclarecido para pesquisas.

Os dados foram analisados de forma descritiva e comparativa, seguida de análise inferencial. O teste estatístico utilizado foi o de associação do qui-quadrado. O nível de significância adotado foi de $5 \%$. Os resultados foram cruzados e analisados segundo as variáveis: sexo; tipo facial obtido por meio da análise cefalométrica; e tipo facial obtido por meio da análise clínica da face.

\section{RESULTADOS}

De acordo com a classificação da face obtida a partir da cefalometria, o tipo facial mais frequente no sexo masculino foi o braquifacial, seguido do mesofacial e, em menor frequência, o dolicofacial. Para o sexo feminino, frequências similares foram encontradas para os tipos meso e braquifacial, mas com valor maior para o mesofacial. Em menor número, foi classificado o tipo dolicofacial. 
Com relação à classificação obtida por meio da análise facial, os tipos faciais encontrados, em ambos os sexos, do mais para o menos frequente foram: face média, curta e longa.

$\mathrm{Na}$ Tabela 1, foram apresentados os dados referentes ao sexo masculino. Nesse caso, não foi possível a realização de testes estatísticos, pois existe uma casela com valor igual a zero. Nesse caso, o valor do nível de significância obtido a partir do teste qui-quadrado não é considerado válido.

Os dados que dizem respeito ao sexo feminino, foram apresentados na Tabela 2. O nível de significância obtido $(p=0,036)$ significa que houve associação estatisticamente significante entre as duas classificações do tipo de face, a partir da cefalometria e da análise facial.

Tabela 1 - Tipo de face determinado pela cefalometria e análise facial, no sexo masculino

\begin{tabular}{lcccccccc}
\hline \multirow{2}{*}{$\begin{array}{l}\text { Tipo facial } \\
\text { (cefalometria) }\end{array}$} & \multicolumn{9}{c}{ Tipo facial (análise facial) } & \multicolumn{2}{c}{ Total } \\
\cline { 2 - 9 } & $\mathbf{N}$ & $\%$ & $\mathbf{N}$ & $\%$ & $\mathbf{N}$ & $\%$ & $\mathbf{N}$ & $\%$ \\
\hline Dolicofacial & $\mathbf{1}$ & $\mathbf{3 3 , 3}$ & 4 & 22,2 & 2 & 15,4 & 7 & 20,5 \\
Mesofacial & 2 & 66,7 & $\mathbf{5}$ & $\mathbf{2 7 , 8}$ & 4 & 30,8 & 11 & 32,4 \\
Braquifacial & 0 & 0,0 & 9 & 50,0 & $\mathbf{7}$ & $\mathbf{5 3 , 8}$ & 16 & 47,1 \\
\hline Total & $\mathbf{3}$ & $\mathbf{8 , 8}$ & $\mathbf{1 8}$ & $\mathbf{5 3 , 0}$ & $\mathbf{1 3}$ & $\mathbf{3 8 , 2}$ & $\mathbf{3 4}$ & $\mathbf{1 0 0 , 0}$ \\
\hline
\end{tabular}

$\mathrm{N}$ - número de sujeitos

Tabela 2 - Tipo de face determinado pela cefalometria e análise facial, no sexo feminino

\begin{tabular}{lcccccccc}
\hline \multirow{2}{*}{$\begin{array}{l}\text { Tipo facial } \\
\text { (cefalometria) }\end{array}$} & \multicolumn{9}{c}{ Longa } & \multicolumn{2}{c}{ Média } & \multicolumn{2}{c}{ Curta } & \multicolumn{2}{c}{ Total } \\
\cline { 2 - 9 } & $\mathbf{N}$ & $\%$ & $\mathbf{N}$ & $\%$ & $\mathbf{N}$ & $\%$ & $\mathbf{N}$ & $\%$ \\
\hline Dolicofacial & $\mathbf{5}$ & $\mathbf{3 5 , 7}$ & 11 & 28,9 & 4 & 21,1 & 20 & 28,2 \\
Mesofacial & 7 & 50,0 & $\mathbf{1 6}$ & $\mathbf{4 2 , 2}$ & 3 & 15,8 & 26 & 36,6 \\
Braquifacial & 2 & 14,3 & 11 & 28,9 & $\mathbf{1 2}$ & $\mathbf{6 3 , 1}$ & 25 & 35,2 \\
\hline Total & $\mathbf{1 4}$ & $\mathbf{1 9 , 7}$ & $\mathbf{3 8}$ & $\mathbf{5 3 , 5}$ & $\mathbf{1 9}$ & $\mathbf{2 6 , 8}$ & $\mathbf{7 1}$ & $\mathbf{1 0 0 , 0}$ \\
\hline
\end{tabular}

$(p)=0,036$ *

* Nível de significância estatística segundo o teste de associação do qui-quadrado

$\mathrm{N}$ - número de sujeitos

\section{DISCUSSÃO}

No geral, foi observado que a frequência dos tipos faciais baseada na classificação obtida por meio da análise cefalométrica de Ricketts seguiu a ordem decrescente: braquifacial, dolicofacial e mesofacial. Foi encontrada ordem decrescente semelhante em outros dois estudos, ou seja, dolicofacial e mesofacial ${ }^{10,11}$. Entretanto, outras pesquisas, apresentaram ordem decrescente diferente: mesofacial, dolicofacial e braquifacial ${ }^{12-14}$; dolicofacial, mesofacial e braquifacial ${ }^{15,16}$; dolicofacial, braquifacial e mesofacial ${ }^{2,17}$; mesofacial e dolicofacial ${ }^{18}$; mesofacial, braquifacial e dolicofacial ${ }^{19-21}$.

Deve-se ressaltar que não existe na literatura pesquisada uma causa que relacione tipologia facial e sua incidência, até porque há grande variabilidade entre os testes utilizados para determinação do tipo de face. Por esse motivo, muitas vezes uma classificação a partir de um teste pode ter resultado diferente ao se utilizar outra avaliação, como comprovado em algumas pesquisas ${ }^{12,22}$.

No que diz respeito à análise facial, vários autores apresentaram, a partir de seus estudos, sua importância para o diagnóstico do clínico ${ }^{5,7,23-29}$. Sugere-se que para a análise facial ser considerada confiável é necessário que os seguintes aspectos sejam aferidos na posição frontal e de perfil: rosto, feições, simetria, harmonia e proporções faciais; tendência a qual tipo de face; altura e largura facial; terços faciais, em especial o inferior; largura bizigomática; contorno e grau de curvatura do perfil; formato do crânio e da cabeça; linha do cabelo; testa; olhos; sobrancelhas; orelhas; maxila, forma e altura da mandíbula, largura bigoníaca, contorno do queixo; lábios, língua, boca, dentes, linha média, 
posição do filtro, oclusão e sorriso; bochechas; nariz, ângulo nasolabial, base e projeção nasal; linha queixo-pescoço; músculos, expressões faciais e marcas de expressão 5,23,25,29.

Nesse tipo de análise, deve-se levar em conta que a face é uma particularidade de cada um e não existe uma exatamente igual à outra. A análise deve considerar o sexo, os traços familiares, a tipologia facial, a raça e a etnia ${ }^{23}$.

O fonoaudiólogo é um dos profissionais que realiza a análise facial em seus pacientes ao avaliar suas estruturas orofaciais, em que se abordam os aspectos morfológicos, posturais e funcionais. Além disso, acredita-se que geralmente faz parte da avaliação fonoaudiológica a definição do tipo de face a partir dos achados clínicos e da correlação com as características gerais de cada tipo.

Esse dado foi considerado nesta pesquisa, mas, nesse caso, a classificação do tipo facial foi apenas por meio da análise visual e indireta da face. Por essa razão, o devido cuidado foi tomado na escolha dos juízes, que além de especialistas em motricidade orofacial deveriam ter experiência mínima de cinco anos na área. Os juízes ficaram à vontade no que diz respeito à análise da face. Dessa forma, não se aconselhou como ou o que analisar em cada face para não induzir os resultados.

Foi observado que os juízes apontaram como mais frequente o tipo de face média, seguido da face curta e da longa. Acredita-se que esses achados decorrem do fato de que provavelmente os sujeitos participantes não apresentavam faces muito discrepantes, já que os casos cirúrgicos não foram incluídos. Deve-se pontuar também que geralmente a avaliação do paciente na clínica fonoaudiológica não é apenas visual e outros aspectos são considerados, como as funções estomatognáticas e o tônus muscular.

Além da presença de histórico de cirurgia ortognática, outro critério de exclusão adotado foi ser da raça negra ou oriental, pois existe uma variação do formato e configuração das estruturas craniofaciais de acordo com essas raças, como encontrado em alguns estudos ${ }^{30-33}$.

Ao se comparar a classificação do tipo de face por meio da cefalometria com a baseada na análise facial, detectou-se que a maioria dos indivíduos masculinos dolicofaciais foi classificada como face média, ou seja, quatro entre sete sujeitos $(57,14 \%)$. Os 11 mesofaciais foram identificados como face média $(\mathrm{N}=5 ; 45,45 \%)$, curta ( $\mathrm{N}=4 ; 36,36 \%)$ ou longa $(\mathrm{N}=2 ; 19,19 \%)$. Dos 16 braquifaciais, a maioria ( $N=9 ; 56,25 \%)$ foi apontada como face média, e os demais como face curta ( $\mathrm{N}=7 ; 43,75 \%)$.

No sexo feminino, observou-se que 11 dentre 20 , ou seja, $55 \%$ dos indivíduos dolicofaciais foram apontados como face média. Dos 26 sujeitos mesofaciais, a maioria, ( $\mathrm{N}=16 ; 61,53 \%)$ também foram classificados como face média. Com relação aos 25 braquifaciais, $12(48 \%)$ foram identificados como face curta, 11 (44\%) como média e dois (4\%) como longa.

A partir desses achados, observou-se que não houve uma correspondência satisfatória ao se comparar a determinação do tipo de face obtido por meio da análise facial com o detectado por meio da cefalometria. Não foi possível comparar esses achados com dados da literatura, pois não foi encontrado nas referências pesquisadas nenhum estudo em que se classificou o tipo de face apenas por meio da análise facial, seja essa análise realizada de forma direta ou indireta.

A avaliação dos tecidos moles é essencial ao se buscar a estética e o equilíbrio facial. Caso esses aspectos não sejam considerados, a face pode se tornar mais ou menos aceitável esteticamente, tanto para profissionais como para leigos. Por essa razão, a análise cefalométrica não deve ser utilizada de forma isolada. É importante analisar a estética facial para diagnóstico, planejamento e tratamento ortodôntico e/ou cirúrgico 4,5,8,17,20.

Entretanto, a análise facial direta ou por meio de fotografias deve ser associada à análise radiográfica da face ${ }^{6}$. Mas, a marcação dos pontos utilizados como referência na cefalometria é subjetiva e passível de erros, o que pode acarretar em resultados enganosos ${ }^{34}$. Por esse motivo, as duas formas de análise, por meio da cefalometria e direta ou a partir de fotografias, devem ser utilizadas conjuntamente.

Dessa forma, acredita-se que, para a determinação do tipo facial, a análise facial, preferencialmente realizada de forma direta, deve ser mais uma ferramenta a contribuir com o diagnóstico fonoaudiológico de cada caso. Todavia, não deve ser utilizada de forma isolada. É fundamental que exames complementares, como a cefalometria, e a discussão do caso com os profissionais afins façam parte do processo de avaliação e do planejamento terapêutico.

\section{CONCLUSÃO}

A análise facial para identificação do tipo facial realizada por meio de fotografias padronizadas não foi considerada confiável, quando utilizada isoladamente, ao ser comparada com a classificação do tipo de face obtido a partir da análise cefalométrica.

\section{AGRADECIMENTOS}

À CAPES, pelo auxílio financeiro e pelo incentivo à pesquisa. 


\section{ABSTRACT}

Purpose: to correlate the cephalometrical findings with the ones from facial analysis performed by means of photographs to classify facial type, according to the gender. Methods: 105 leukoderm adults took part, 34 male (32.4\%) and 71 female (67.6\%) with ages between 20 and 40 years old, in a private orthodontic clinic in Belo Horizonte, MG - Brazil. The cephalometrics analysis findings for determining facial type from the Ricketts VERT index were compared with the facial classification performed by means of clinical facial analysis based on observation of two photographs. For this analysis, three Speech and Language Pathologists specialized in orofacial myology were involved. Results: there was no satisfactory correspondence when comparing the two forms of facial type classification presented in this study. Most of the dolichofacial males were classified as medium face by means of facial analysis, meaning four among seven subjects $(57.14 \%)$. The eleven mesofacial subjects were identified as medium face $(\mathrm{N}=5 ; 45.45 \%)$ or short $(\mathrm{N}=4 ; 36.36 \%)$ and the 16 braquifacial subjects as medium face $(\mathrm{N}=9 ; 56.25 \%)$ or short $(\mathrm{N}=7 ; 43.75 \%)$. With females, it was observed that 11 among 20, meaning 55\% of the dolichofacial individuals were appointed as medium face. Among the 26 mesofacial subjects, $16(61.53 \%)$ were also classified as medium face. Regarding the 25 braquifacial subjects, $12(48 \%)$ were identified as short face and 11 (44\%), as medium. Conclusion: facial type classification based only on facial analysis performed by means of photographs was not considered trustworthy, if used independently, when compared with cephalometrics analysis findings.

KEYWORDS: Face; Morphology; Adult; Cephalometry; Photography; Comparative Study

\section{REFERÊNCIAS}

1. Bianchini EMG. A cefalometria nas alterações miofuncionais orais: diagnóstico e tratamento fonoaudiológico. 5.ed. Carapicuíba: Pró-Fono; 2002.

2. Santos SH, Moraes LC, Médici Filho E, Castilho JCM, Moraes MEL. Aplicação do método linear e geométrico utilizando radiografias cefalométricas laterais, para diferenciar e identificar a proporção divina em três tipos faciais. Cienc Odontol Bras. 2005; 8(3):10-21.

3. Pereira ACL, Woitchunas CA, Oliveira FAM, Woitchunas GFP, Oliveira MG, Santos SMMC. Cefalometria computadorizada de Ricketts: estudo comparativo, inter-raças e gêneros, de indivíduos com harmonia facial. Rev Ortodontia SPO. 2007; 40(1):14-21.

4. Sant'Ana E, Kuriki EU, Arnett W, Lautenschläger GAC, Yaedu RYF. Avaliação comparativa do padrão de normalidade do perfil facial em pacientes brasileiros leucodermas e em norte-americanos. Dental Press Ortodon Ortop Facial. 2009; 14(1):80-9. 5. Suguino R, Ramos AL, Terada H, Furquim LZ, Maeda L, Silva Filho OG. Análise facial. Dental Press Ortod Ortop Maxilar. 1996; 1(1):86-107.

6. Reche R, Colombo VL, Verona J, Moresca CA, Moro A. Análise do perfil facial em fotografias padronizadas. Dental Press Ortodon Ortop Facial. 2002; 7(1):37-45.

7. Schlickmann ICA, Moro A, Dos Anjos A. Análise do perfil facial masculino adulto jovem, esteticamente agradável, em fotografias padronizadas: comparação da medição manual com a computadorizada. Dental Press Ortodon Ortop Facial. 2008; 13(6):98-107.

8. Feres R, Vasconcelos MHF. Estudo comparativo entre a análise facial subjetiva e a análise cefalométrica de tecidos moles no diagnóstico ortodôntico. Dental Press Ortodon Ortop Facial. 2009; 14(2):81-8.

9. Ricketts RM. Orthodontic diagnosis and planningtheir roles in preventive and rehabilitative dentristy. Denver: Rocky Mountain Orthodontics; 1982. 269p.

10. Pereira AC, Jorge TM, Ribeiro Júnior PD, Berretin-Felix G. Características das funções orais de indivíduos com má oclusão classe III e diferentes tipos faciais. Dental Press Ortodon Ortop Facial. 2005; 10(6):111-9.

11. Sies ML, Farias SR, Vieira MM. Respiração oral: relação entre o tipo facial e a oclusão dentária em adolescentes. Rev Soc Bras Fonoaudiol. 2007; 12(3):191-8.

12. Moresca R, Reis SAB, Vigorito JW, Scanavini MA. Estudo comparativo cefalométrico-radiográfico do padrão facial na má-oclusão de classe II, 1a de Angle, empregando as análises cefalométricas de Ricketts e Siriwat \& Jarabak. J Bras Ortodon Ortop Facial. 2002; 7(42):520-5.

13. Pieri LV, Faltin Júnior K, Ortolani CLF, Faltin RM, Almeida MAA. Crescimento da base craniana nos diferentes tipos faciais nos relacionamentos maxilomandibulares ortopédicos de classe I, II e III: 
parte 1. Dental Press Ortodon Ortop Facial. 2007; 12(1):110-7.

14. Araújo $M C$, Nahás $A C R$, Cotrim-Ferreira FA, Carvalho PEG. Estudo cefalométrico da correlação da anatomia da base craniana com o padrão facial e as bases apicais. Dental Press Ortodon Ortop Facial. 2008; 13(4):67-76.

15. Bianchini AP, Guedes ZCF, Vieira MM. Estudo da relação entre a respiração oral e o tipo facial. Rev Bras Otorrinolaringol. 2007; 73(4):500-5.

16. Queiroz KL, Wassall T, Miyamura ZY, Arsati YBOL, Loducca FE. Avaliação do ângulo articular nos diferentes tipos faciais- em crianças brasileiras leucodermas (dos 08 aos 10 anos). RGO. 2003; 51(4):231-6.

17. Arslan SG, Genç C, Odabaş B, Kama JD. Comparison of facial proportions and anthropometric norms among Turkish young adults with different face types. Aesthetic Plast Surg. 2008; 32(2):234-42. 18. Daenecke S, Bianchini EMG, Silva APBV. Medidas antropométricas de comprimento de lábio superior e filtro. Pró-Fono. 2006; 18(3):249-58.

19. Canuto MSB, Assis RS, Gouveia PMP, Nemr K. Análise comparativa entre presença de diastemas e tipos faciais. Rev CEFAC. 2006; 8(2):162-70.

20. Silva Filho OG, Herkrath FJ, Queiroz APC, Aiello CA. Padrão facial na dentadura decídua: estudo epidemiológico. Dental Press Ortodon Ortop Facial. 2008; 13(4):45-59.

21. Vianna-Lara MS, Caria PH, Tosello DO, Lara F, Amorim MM. Electromyographic activity of masseter and temporal muscles with different facial types. Angle Orthod. 2009; 79(3):515-20.

22. Scanavini C, Vigorito JW. Estudo cefalométricoradiográfico das possíveis correlações existentes entre as análises de Vigorito, Ricketts e Siriwat \& Jarabak na definição dos tipos faciais, em indivíduos leucodermas. Ortodontia. 2001; 34(3):27-41.

23. Enlow DH, Poston WR, Bakor SF. Crescimento facial. 3. ed. São Paulo: Artes Médicas; 1993.

24. Edler R, Agarwal P, Weitheim D, Greenhill D. The use of anthropometric proportion indices in the measurement of facial attractiveness. Eur $\mathrm{J}$ Orthod. 2006; 28(3):274-81.

RECEBIDO EM: 31/03/2009

ACEITO EM: 14/09/2009

Endereço para correspondência:

Rossana Ribeiro Ramires

Rua Teopompo de Vasconcelos, 375/ 163

São José dos Campos - SP

CEP: 12243-830

E-mail: rossana_ramires@yahoo.com.br
25. Reis SAB, Abrão J, Capelozza Filho L, Claro CAA. Análise facial subjetiva. Dental Press Ortodon Ortop Facial. 2006; 11(5):159-72.

26. Reis SAB, Abrão J, Capelozza Filho L, Claro CAA. Análise facial numérica do perfil de brasileiros padrão I. Dental Press Ortodon Facial. 2006; 11(6):24-34.

27. Tôrres SC, Costa C, Faltin Junior K. Estudo da posição natural da cabeça em relação ao plano horizontal de Frankfurt na avaliação mandibular de indivíduos com padrão facial de classe I e classe II. Dental Press Ortodon Ortop Facial. 2006; 11(1):84-98.

28. Trevisan F, Gil CTLA. Análise fotogramétrica e subjetiva do perfil facial de indivíduos com oclusão normal. Dental Press Ortodon Ortop Facial. 2006; 11(4):24-35.

29. Cattoni DM, Fernandes FD, Di Francesco RC, Latorre MRDO. Características do sistema estomatognático de crianças respiradoras orais: enfoque antroposcópico. Pró-Fono. 2007; 19(4):347-51.

30. Farkas LG, Katic MJ, Forrest CR, Alt KW, Bagic I, Baltadjiev G, et al. International anthropometric study of facial morphology in various ethnic groups/ races. J Craniofac Surg. 2005; 16(4):615-46.

31. Farkas LG, Katic MJ, Forrest CR. Comparison of craniofacial measurements of young adult AfricanAmerican and North American white males and females. Ann Plast Surg. 2007; 59(6):692-8.

32. Hajniš K, Farkas LG, Ngim RCK, Lee ST, Venkatadri G. Racial and ethnic morphometric differences in the craniofacial complex. In: Farkas LG. Anthropometry of the head and face. 2. ed. New York: Raven Press; 1994. p.201-18.

33. Parro FM, Toledo MR, Gomes ICD, Marchesan IQ. Diferenças antropométricas entre mulheres brancas e negras após crescimento puberal. Rev CEFAC. 2005; 7(4):459-65.

34. Grossi VCC, Mazzieiro ET, Siqueira VCV. Estudo comparativo entre avaliações esqueléticas e do perfil tegumentar utilizadas no diagnóstico ortodôntico sagital. Dental Press Ortodon Ortop Facial. 2007; 12(4):107-16. 